Brief Communication

\title{
Stbd1 is highly elevated in skeletal muscle of Pompe disease mice but suppression of its expression does not affect lysosomal glycogen accumulation
}

\author{
Haiqing Yi, Keri B. Fredrickson, Stuti Das, Priya S. Kishnani, Baodong Sun * \\ Division of Medical Genetics, Department of Pediatrics, Duke University Medical Center, Durham, NC, USA
}

\section{A R T I C L E I N F O}

\section{Article history:}

Received 7 May 2013

Received in revised form 8 May 2013

Accepted 9 May 2013

Available online 18 May 2013

\section{Keywords:}

Stbd1

AAV

ShRNA

Pompe

Lysosomal glycogen

\begin{abstract}
A B S T R A C T
Previous studies strongly suggest that starch binding domain containing protein 1 (Stbd1) plays an important role in intracellular glycogen trafficking into lysosomes. We report here that Stbd1 expression is markedly increased in skeletal muscles but not in heart and liver of GAA-KO mice. An AAV2/9 vector expressing a Stbd1-specific shRNA effectively suppressed Stbd1 expression but did not alter lysosomal glycogen accumulation in the affected tissues of GAA-KO mice. Our results indicate that inhibition of Stbd1 does not appear to be an effective therapeutic approach for Pompe disease.
\end{abstract}

(c) 2013 Elsevier Inc. All rights reserved.

\section{Introduction}

Pompe disease (GSD II) is an autosomal recessive disorder caused by deficiency of the lysosomal enzyme acid- $\alpha$-glucosidase (GAA, EC 3.2.1.20), leading to accumulation of lysosomal glycogen and enlargement of lysosomes in heart, skeletal muscle, and the central nervous system. Patients with the most severe infantile form normally die within the first year of life, while in the milder late-onset forms, muscle weakness is the primary symptom and respiratory failure is the major cause of significant morbidity and mortality [1]. Enzyme replacement therapy (ERT) with recombinant human GAA (rhGAA, alglucosidase alfa) is currently the only available therapy for Pompe disease with remarkable success, but also with significant limitations including the high cost for life-long administration of high-dose enzyme, poor clinical outcomes caused by the formation of high-titer anti-rhGAA antibodies in the majority of patients, and reduced efficacy in the skeletal muscle [2-5]. Thus, there is an unmet need to develop improved therapies for Pompe disease.

Prevention of cytoplasmic glycogen trafficking into lysosome could also be a feasible treatment approach for Pompe disease. Starch binding domain containing protein 1 (Stbd1, also known as GENX-3414 or

Abbreviations: Stbd1, starch binding domain containing protein 1; shRNA, small hairpin RNA; AAV, adeno-associated virus; GSD, glycogen storage disease; GAA, acid $\alpha$-glucosidase; LAMP2, lysosomal associated membrane protein 2; LC3, microtubule-associated protein light chain 3; PYGM, muscle glycogen phosphorylase; EDL, extensor digitorum longus; GAPDH, glyceraldehyde 3-phosphate dehydrogenase.

* Corresponding author at: Division of Medical Genetics, Department of Pediatrics, Duke University Medical Center, 595 Lasalle Street, GSRB1 Building, 4th Floor, Room 4044, PO Box DUMC 103856, Durham, NC 27710, USA. Fax: + 19196840923.

E-mail address: baodong.sun@duke.edu (B. Sun). genethonin 1 ) is a protein with a predicated structure containing a hydrophobic $\mathrm{N}$ terminus and a C-terminal CBM20 glycan binding domain [6,7]. Stbd1 is highly expressed in muscle and liver, the major tissues of glycogen storage $[6,8]$. The function of Stbd1 was unknown but recent studies demonstrated that it is involved in glycogen metabolism $[8,9]$. In mice, genetic deletion of muscle glycogen synthase caused a complete loss of Stbd1 protein expression in muscle while liver glycogen synthase knockout led to a $40 \%$ reduction of Stbd1 [8]. In cultured cells, Stbd1 locates to perinuclear compartments and co-localized with glycogen, the lysosomal marker LAMP1 but not the autophagic marker LC3 [8]. These results strongly suggest that Stbd1 is a specific mediator for cytoplasmic glycogen transport into the lysosome and could potentially be a new therapeutic target for Pompe disease. In this report, we aimed to determine whether inhibition of Stbd1 will block lysosomal glycogen transport, and thereby prevent glycogen accumulation in Pompe disease.

\section{Materials and methods}

\subsection{AAV vector construction and preparation}

A 1.08-kb fragment containing the shRNA that targets mouse Stbd1 mRNA site AGCTTTAAGTTATAACTGA (nt 1938 to1956, NM_175096) was PCR amplified from PGIPZ vector clone V3LMM_521381 (Open Biosystems, Huntsville, AL) using Pfu DNA polymerase (Stratagene, La Jolla, CA), and cloned into pAAV-CBhGAApA to replace the hGAA cDNA [10]. The shRNA expression in the resulting plasmid, pAAV-CBshRNApA, is driven by the hybrid CMV enhancer/chicken $\beta$-actin $(C B)$ promoter. AAV2/9 vector was produced in HEK 293T cells using standard method and purified using iodixanol gradient ultracentrifugation [11,12]. The 
AAV packaging plasmid pRep2Cap9 was kindly provided by Dr. James M. Wilson, University of Pennsylvania.

\subsection{Stbd1 knock-down in GAA-KO mice}

For systematic knock-down, the AAV vector at a dose of $1 \times 10^{12}$ viral genomes (vg) per mouse or PBS was intravenously administered to one-month-old GAA-knockout (GAA-KO) mice generated on a C57 genetic background [13], and tissues were collected 60 days later. For muscle-specific knock-down, $2 \times 10^{11} \mathrm{vg} /$ mouse vector was intramuscularly injected to the right gastrocnemius of 10-day-old mice and both right and left gastrocnemius were collected 30 days later. All animal procedures were done in accordance with Duke University Institutional Animal Care and Use Committee-approved guidelines.

\subsection{Quantitation of Stbd1 mRNA}

Total RNA extraction and reverse transcription were performed as described [14]. Real-time PCR was performed using LightCycler 480 (Roche Diagnostics, Indianapolis, IN) and primers 5'-TGAGCCCTGA ACCTTCCGATC-3' (forward) and 5'-GTGCTGTCGTCCATTCTCTGA-3' (reverse). $\beta$-actin was used as internal control [14].

\subsection{Western blot}

RIPA extracts of tissues were analyzed by Western blotting [14]. Primary antibodies used: rabbit anti mouse Stbd1, gift from Dr. Peter J. Roach, Indiana University [8]; LAMP2 and rabbit anti PYGM (Abcam); rabbit anti glycogen synthase (Cell Signaling); goat anti GAPDH (Novus Biologicals); rabbit anti LC3B; and HRP-conjugated mouse anti $\beta$-actin (Sigma-Aldrich).

\subsection{Histology and glycogen measurement}

Periodic acid-Schiff (PAS) staining and glycogen content assay were performed as described [15].

\subsection{Statistical analyses}

Data were presented as mean \pm standard deviation. The significance of differences was assessed using two-tailed, equal variance student $t$-test. $\mathrm{p}<0.05$ was considered significant.

\section{Results}

\subsection{Tissue distribution of Stbd1 in GAA-KO and wild-type mice}

Western blot showed that Stbd1expression is most abundant in skeletal muscles (quadriceps and gastrocnemius) of 3-month-old GAA-KO mice; the protein is apparently less in liver, and weakly detectable in heart (Fig. 1A). We then compared Stbd1 expression in the GAA-KO mice with age-matched wild-type (C57BL/6) mice. Stbd1 was markedly higher in GAA-KO than in wild-type mice in both quadriceps and gastrocnemius muscles (Fig. 1B). There was no noticeable difference in liver or heart between the two. Interestingly, both GAA-KO and wild-type mice expressed more Stbd1 in fast-twitch extensor digitorum longus (EDL) muscles (predominantly type IIB fibers) than in slow-twitch soleus muscles (predominantly type I fiber) (Fig. 1C).

\section{2. shRNA mediated Stbd1 knockdown in GAA-KO mice}

To investigate whether reduction of Stbd1 expression in GAA-KO mice would prevent lysosomal glycogen accumulation, an AAV2/9
A

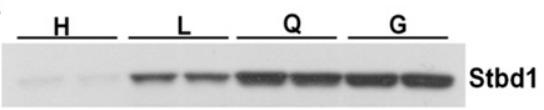

B

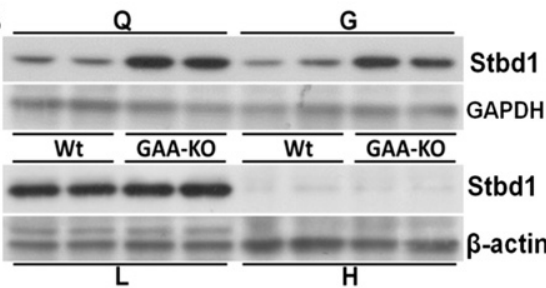

C

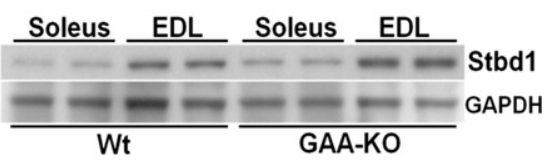

D

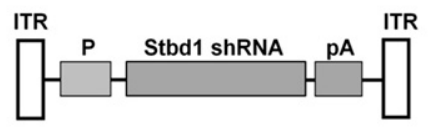

E

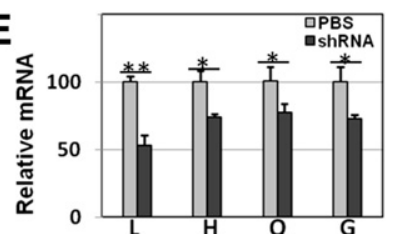

$\mathbf{F}$

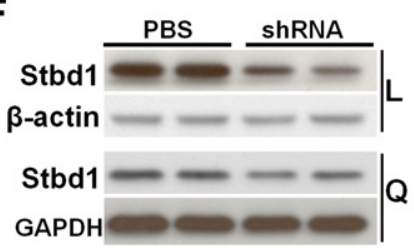

G

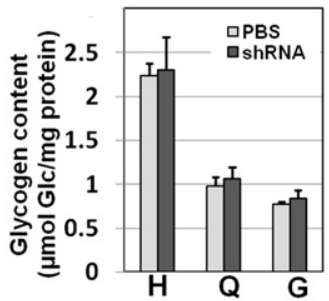

H
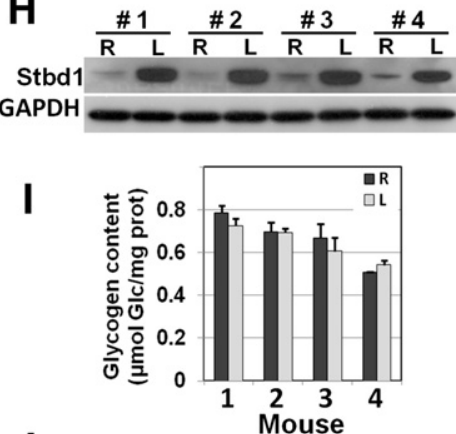

$\mathbf{J}$

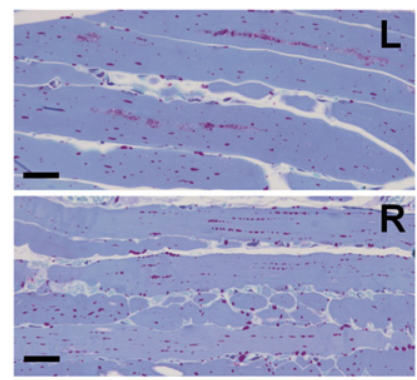

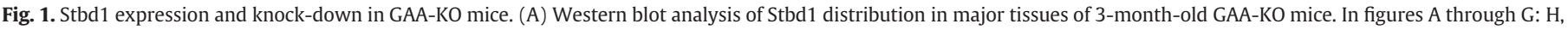

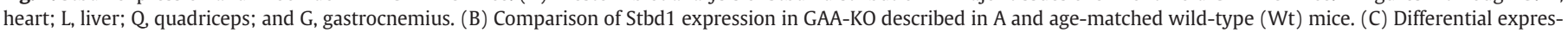

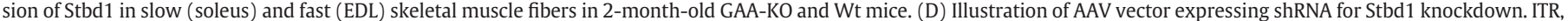

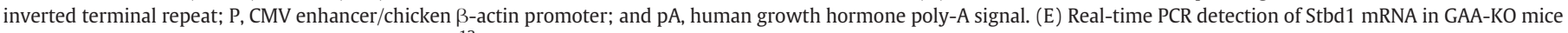

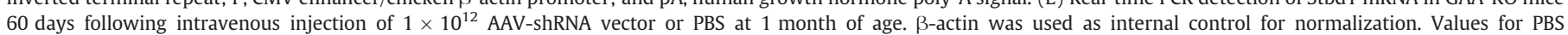

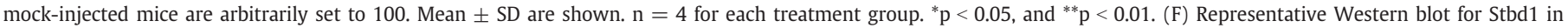

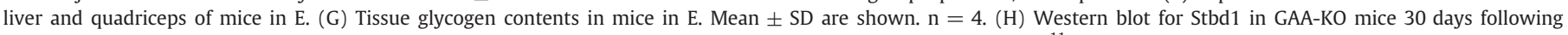

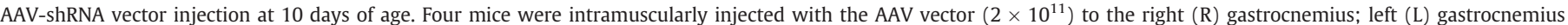

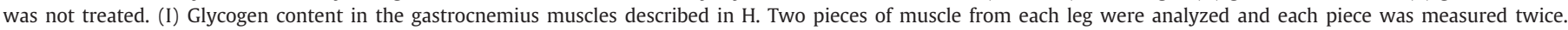

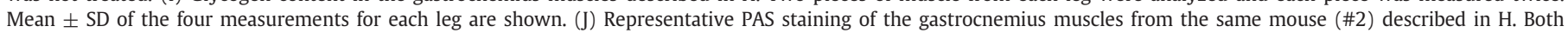
treated-muscle $(\mathrm{R})$ and untreated-muscle $(\mathrm{L})$ showed a similar degree of PAS positive lysosomal glycogen. Magnification $=400 \times$, and scale bars $=25 \mu \mathrm{m}$. 
vector expressing Stbd1-specific shRNA (Fig. 1D) was intravenously injected into 1 -month-old GAA-KO mice $\left(1 \times 10^{12} \mathrm{vg} /\right.$ mouse $)$. After 60 days of AAV-shRNA treatment, Stbd 1 mRNA was reduced by $47 \%$ in liver, $27 \%$ in heart, $23 \%$ in quadriceps, and $28 \%$ in gastrocnemius (Fig. 1E). The Stbd1 protein levels were also markedly reduced in liver and skeletal muscle (Fig. 1F). There was no obvious reduction in the heart (not shown) possibly due to the low expression level. However, glycogen content was not affected by the shRNA treatment in heart and skeletal muscle (Fig. $1 \mathrm{G}$ ). To confirm this result, $2 \times 10^{11}$ of the AAV-shRNA vector was intramuscularly administrated to the right gastrocnemius of 10-day-old GAA-KO mice $(n=4)$. After 30 days, Stbd1 protein was nearly eliminated in the injected leg in all 4 mice (Fig. $1 \mathrm{H}$ ), but the glycogen content was unchanged compared with the counterpart leg of the same mouse (Fig. 1I). PAS staining showed a similar pattern of lysosomal glycogen distribution between the injected and the control legs (Fig. 1J). Inhibition of Stbd1 expression in muscle had no effect on the expression of glycogen synthase, glycogen phosphorylase, LC3 (autophagy marker), and LAMP2 (lysosome marker), as determined by Western blot (not shown).

\section{Discussion}

Lysosomal glycogen degradation plays an important role in overall cellular glycogen metabolism because deficiency in the lysosomal glycogen degrading enzyme GAA causes Pompe disease. Understanding the mechanism of how glycogen enters the lysosome is a critical step in finding a new therapeutic target for Pompe disease. In GAA-KO mice, autophagy was dramatically induced in both slow- and fast-twitch skeletal muscles [16]. However, suppression of autophagy by inactivating a critical autophagy gene Atg7 reduced glycogen content only by $50-60 \%$ in fast muscle, and had no effect on slow and cardiac muscles of GAA-KO mice [17]. These studies strongly implied that other mechanisms than autophagy exist for lysosomal glycogen trafficking.

A previous study strongly suggests that Stbd1 is a specific mediator for cytoplasmic glycogen transport into the lysosome via a non-classic glycogen autophagy pathway [8]. In this study, we observed that Stbd1 expression was markedly elevated in skeletal muscles but not in liver and heart of GAA-KO mice when compared with age-matched wild-type mice (Fig. 1B). We also found that Stbd1 expression was higher in fast muscles (quadriceps, gastrocnemius, and EDL) than in slow (soleus) and cardiac muscles of GAA-KO mice (Figs. 1A \& C). A similar pattern was also observed in wild-type mice (Fig. 1C). This suggests a unique role of Stbd1 in fast-twitch skeletal muscle. In wild-type mice, glycogen is invisible by PAS staining and glycogen content is almost undetectable by the standard biochemical assay in the skeletal and cardiac muscles; in GAA-KO mice, the high level of glycogen content in these muscles is attributed to the accumulation of glycogen in the lysosomes $[13,18]$. Although an early study strongly suggested that Stbd1 is a mediator of lysosomal glycogen trafficking, our results showed that suppression of Stbd1 expression by AAV-shRNA treatment did not change lysosomal glycogen accumulation in GAA-KO mice (Figs. 1G, I, \&J). This indicates that either Stbd1 is not involved in lysosomal glycogen transport, or the AAV-shRNA mediated gene silencing was not sufficient to block its function in this process.

In summary, Stbd1 knockdown does not appear to be a suitable therapeutic approach for Pompe disease. The function of Stbd1 is still unclear. We are developing a Stbd1knockout mouse model to further investigate its role in glycogen metabolism.

\section{Conflict of interest}

The authors declare no conflict of interest.

\section{Acknowledgments}

We thank Dr. Beth L. Thurberg for her help with the histology. We are grateful to Dr. Peter J. for providing us with the anti-mouse Stbd1 antibody.

\section{References}

[1] Y.T. Chen, P.S. Kishnani, D.D. Koeberl, Glycogen storage diseases, in: D. Valle, A Beaudet, B. Vogelstein, K. Kinzler, S. Antonarakis, A. Ballabio (Eds.), Scriver's Online Metabolic \& Molecular Bases of Inherited Disease, McGraw-Hill, New York, 2009.

[2] N. Raben, M. Danon, A.L. Gilbert, S. Dwivedi, B. Collins, B.L. Thurberg, R.J. Mattaliano, K. Nagaraju, P.H. Plotz, Enzyme replacement therapy in the mouse model of Pompe disease, Mol. Genet. Metab. 80 (2003) 159-169.

[3] A. Amalfitano, A.R. Bengur, R.P. Morse, J.M. Majure, L.E. Case, D.L. Veerling, J. Mackey, P. Kishnani, W. Smith, A. McVie-Wylie, J.A. Sullivan, G.E. Hoganson, J.A. Phillips III, G.B. Schaefer, J. Charrow, R.E. Ware, E.H. Bossen, Y.T. Chen, Recombinant human acid alpha-glucosidase enzyme therapy for infantile glycogen storage disease type II: results of a phase I/II clinical trial, Genet. Med. 3 (2001) $132-138$.

[4] M. Nicolino, B. Byrne, J.E. Wraith, N. Leslie, H. Mandel, D.R. Freyer, G.L. Arnold, E.K Pivnick, C.J. Ottinger, P.H. Robinson, J.C. Loo, M. Smitka, P. Jardine, L. Tato, B. Chabrol, S. McCandless, S. Kimura, L. Mehta, D. Bali, A. Skrinar, C. Morgan, L. Rangachari, D. Corzo, P.S. Kishnani, Clinical outcomes after long-term treatment with alglucosidase alfa in infants and children with advanced Pompe disease, Genet. Med. 11 (2009) 210-219.

[5] P.S. Kishnani, P.C. Goldenberg, S.L. DeArmey, J. Heller, D. Benjamin, S. Young, D. Bali, S.A. Smith, J.S. Li, H. Mandel, D. Koeberl, A. Rosenberg, Y.T. Chen, Cross-reactive immunologic material status affects treatment outcomes in Pompe disease infants, Mol. Genet. Metab. 99 (2010) 26-33.

[6] S. Bouju, M.F. Lignon, G. Pietu, M. Le Cunff, J.J. Leger, C. Auffray, C.A. Dechesne, Molecular cloning and functional expression of a novel human gene encoding two 41-43 kDa skeletal muscle internal membrane proteins, Biochem. J. 335 (Pt 3) (1998) 549-556.

[7] S. Janecek, A motif of a microbial starch-binding domain found in human genethonin, Bioinformatics 18 (2002) 1534-1537.

[8] S. Jiang, B. Heller, V.S. Tagliabracci, L. Zhai, J.M. Irimia, A.A. DePaoli-Roach, C.D Wells, A.V. Skurat, P.J. Roach, Starch binding domain-containing protein $1 /$ genethonin 1 is a novel participant in glycogen metabolism, J. Biol. Chem. 285 (2010) 34960-34971.

[9] D. Stapleton, C. Nelson, K. Parsawar, D. McClain, R. Gilbert-Wilson, E. Barker, B. Rudd K. Brown, W. Hendrix, P. O'Donnell, G. Parker, Analysis of hepatic glycogen-associated proteins, Proteomics 10 (2010) 2320-2329.

[10] B.D. Sun, Y.T. Chen, A. Bird, A. Amalfitano, D.D. Koeberl, Long-term correction of glycogen storage disease type II with a hybrid Ad-AAV vector, Mol. Ther. 7 (2003) 193-201.

[11] B. Sun, S.P. Young, P. Li, C. Di, T. Brown, M.Z. Salva, S. Li, A. Bird, Z. Yan, R. Auten, S.D. Hauschka, D.D. Koeberl, Correction of multiple striated muscles in murine Pompe disease through adeno-associated virus-mediated gene therapy, Mol. Ther. 16 (2008) 1366-1371.

[12] W.T. Hermens, O. ter Brake, P.A. Dijkhuizen, M.A. Sonnemans, D. Grimm, J.A Kleinschmidt, J. Verhaagen, Purification of recombinant adeno-associated virus by iodixanol gradient ultracentrifugation allows rapid and reproducible preparation of vector stocks for gene transfer in the nervous system, Hum. Gene Ther. 10 (1999) 1885-1891.

[13] N. Raben, K. Nagaraju, E. Lee, P. Kessler, B. Byrne, L. Lee, M. LaMarca, C. King, J Ward, B. Sauer, P. Plotz, Targeted disruption of the acid alpha-glucosidase gene in mice causes an illness with critical features of both infantile and adult human glycogen storage disease type II, J. Biol. Chem. 273 (1998) 19086-19092.

[14] B. Sun, H. Zhang, L.M. Franco, S.P. Young, A. Schneider, A. Bird, A. Amalfitano, Y.T. Chen, D.D. Koeberl, Efficacy of an adeno-associated virus 8-pseudotyped vector in glycogen storage disease type II, Mol. Ther. 11 (2005) 57-65.

[15] H. Yi, B.L. Thurberg, S. Curtis, S. Austin, J. Fyfe, D.D. Koeberl, P.S. Kishnani, B. Sun Characterization of a canine model of glycogen storage disease type IIla, Dis. Model Mech. 5 (2012) 804-811.

[16] N. Raben, V. Hill, L. Shea, S. Takikita, R. Baum, N. Mizushima, E. Ralston, P. Plotz, Suppression of autophagy in skeletal muscle uncovers the accumulation of ubiquitinated proteins and their potential role in muscle damage in Pompe disease, Hum. Mol. Genet. 17 (2008) 3897-3908.

[17] N. Raben, C. Schreiner, R. Baum, S. Takikita, S. Xu, T. Xie, R. Myerowitz, M Komatsu, J.H. Van der Meulen, K. Nagaraju, E. Ralston, P.H. Plotz, Suppression of autophagy permits successful enzyme replacement therapy in a lysosomal storage disorder - murine Pompe disease, Autophagy 6 (2010) 1078-1089.

[18] L.M. Franco, B. Sun, X. Yang, A. Bird, H. Zhang, A. Schneider, T. Brown, S.P. Young, T.M. Clay, A. Amalfitano, Y.T. Chen, D.D. Koeberl, Evasion of immune responses to introduced human acid alpha-glucosidase by liver-restricted expression in glycogen storage disease type II, Mol. Ther. 12 (2005) 876-884. 